\title{
Hysteretic hERG Channel Gating Current Recorded At Physiological Temperature
}

David Kelly Jones ( $\nabla$ davekj@umich.edu )

University of Michigan-Ann Arbor

\section{Research Article}

Keywords: Hysteretic, hERG, physiological temperature, VSD

Posted Date: December 17th, 2021

DOI: https://doi.org/10.21203/rs.3.rs-1149950/v1

License: (a) (i) This work is licensed under a Creative Commons Attribution 4.0 International License. Read Full License

Version of Record: A version of this preprint was published at Scientific Reports on April 8th, 2022. See the published version at https://doi.org/10.1038/s41598-022-10003-7. 


\section{Abstract}

Cardiac hERG channels comprise at least two subunits, hERG 1a and hERG 1b, and drive cardiac action potential repolarization. hERG 1a subunits contain a cytoplasmic PAS domain that is absent in $h E R G 1 b$. The hERG 1a PAS domain regulates voltage sensor domain (VSD) movement, but hERG VSD behavior and its regulation by the hERG 1a PAS domain have not been studied at physiological temperatures. We recorded gating charge from homomeric hERG $1 \mathrm{a}$ and heteromeric $\mathrm{hERG} 1 \mathrm{a} / \mathrm{1b}$ channels at near physiological temperatures $\left(36 \pm 1^{\circ} \mathrm{C}\right)$ using pulse durations comparable in length to the human ventricular action potential. The voltage dependence of deactivation was hyperpolarized relative to activation, reflecting VSD relaxation at positive potentials. These data suggest that relaxation (hysteresis) works to delay pore closure during repolarization. Interestingly, hERG 1a VSD deactivation displayed a double Boltzmann distribution, but hERG 1a/1b deactivation displayed a single Boltzmann. Disabling the hERG1a PAS domain using a PAS-targeting antibody similarly transformed hERG 1a deactivation from a double to a single Boltzmann, highlighting the contribution of the PAS in regulating VSD movement. These data represent, to our knowledge, the first recordings of hERG gating charge at physiological temperature and demonstrate that VSD relaxation (hysteresis) is present in hERG channels at physiological temperature.

\section{Introduction}

The $K C N H 2$ gene encodes the hERG 1 voltage-gated potassium channel that conducts the critical repolarizing current $\mathrm{I}_{\mathrm{Kr}}^{1,2}$. $K C N H 2$ mutations that reduce $\mathrm{I}_{\mathrm{Kr}}$ or off-target hERG 1 channel block cause the cardiac disorder long QT syndrome (LQTS) and increase the likelihood for cardiac arrhythmia and sudden cardiac death ${ }^{2,3}$.

hERG channels are tetrameric with a single, central pore that is gated by the movements of the voltage sensing domain (VSD) of each subunit ${ }^{4,5}$. It is the concerted movement of the VSDs in response to changes in the membrane electric field that triggers pore opening or closure in voltage-dependent channels, including hERG channels ${ }^{6-11}$. Like other voltage-sensitive channels, hERG VSDs transition from their active state to a more energetically favorable "relaxed" state during prolonged depolarization (> 300 ms at room temperature $)^{9,12-15}$. VSD relaxation hyperpolarizes the voltage dependence of VSD deactivation and delays pore closure upon repolarization $11,12,14,16$. Whether VSD relaxation occurs at physiological temperature is unknown, but if present, would presumably work to enhance hERG 1 channel open probability during cardiac repolarization, by delaying pore closure.

Native hERG 1 channels are composed of at least two subunits, hERG $1 \mathrm{a}$ and hERG $1 b^{17-21}$ that are identical with the exception of their respective $\mathrm{N}$-termini ${ }^{19,20}$. The hERG 1a N-terminal domain contains a Per-Arnt-Sim (PAS) domain ${ }^{22}$, which modulates hERG 1 gating through interactions between the cytoplasmic linker of the 4th and 5th transmembrane helices and the C-terminal cyclic nucleotide binding homology domain (CNBHD) ${ }^{23-27}$. Mutations within the hERG 1a PAS domain that disrupt the 
PAS/CNBHD interaction accelerate channel activation and deactivation, and disrupt inactivation ${ }^{23,28-33}$. Disrupting the PAS/CNBHD interaction also accelerates VSD deactivation and decreases the hysteresis of ionic current ${ }^{13,15,16}$. Surprisingly, the reports of PAS disruption on VSD hysteresis are mixed, where two reports demonstrated reduced hysteresis ${ }^{13,15}$, but a third reported that VSD hysteresis remained intact ${ }^{16}$. hERG 1b subunits have a unique N-terminal domain that lacks a PAS domain. Co-expression of the PASdeficient hERG $1 \mathrm{~b}$ subunit with hERG $1 \mathrm{a}$ reduces the relative number of PAS domains per channel, and thus heteromeric hERG 1a/1b channels display accelerated channel kinetics and larger currents compared to hERG 1 a homomeric channels ${ }^{34,35}$. Gating charge from heteromeric hERG $1 \mathrm{a} / 1 \mathrm{~b}$ channels has not been recorded.

Here we report hERG gating charge recorded from hERG $1 \mathrm{a}$ and $\mathrm{hERG} 1 \mathrm{a} / \mathrm{1b}$ channels at near physiological temperatures $\left(36 \pm 1^{\circ} \mathrm{C}\right)$. We found that hERG 1 VSD relaxation is intact in homomeric hERG 1a channels and heteromeric hERG 1a/1b channels at physiological temperatures. Surprisingly, hERG 1a/1b channels displayed enhanced gating charge hysteresis, reflecting a stabilized relaxed state, compared to homomeric hERG 1 a channels. PAS disruption, using a PAS targeting antibody on homomeric channels transformed homomeric hERG 1a gating charge and ionic current to a phenotype similar to that observed in heteromeric hERG $1 \mathrm{a} / 1 \mathrm{~b}$ channels. These data provide a more in-depth mechanism for the differential gating between homomeric hERG 1a channels and heteromeric hERG $1 \mathrm{a} / 1 \mathrm{~b}$ channels. These data also demonstrate that VSD relaxation is intact in hERG 1 channels at physiological temperature.

\section{Results}

\section{hERG 1a Gating Current at Physiological Temperature}

In this study, we sought to characterize hERG 1 hysteresis at physiological temperatures $\left(36 \pm 1^{\circ} \mathrm{C}\right)$. We first measured hERG 1a gating current activation and deactivation from HEK293 cells stably expressing the hERG 1a subunit (Fig. 1). To isolate the hERG 1 gating current from ionic current, we replaced the monovalent cations of the bath and pipette solutions with $\mathrm{NMDG}^{+}$and $\mathrm{TEA}^{+}$, respectively. We measured the voltage dependence of VSD activation by recording the gating current elicited during a $400 \mathrm{~ms}$ test potential from a $-100 \mathrm{mV}$ holding potential (Fig. 1a). We measured the voltage dependence of VSD deactivation by recording the gating current elicited during a 400 ms hyperpolarizing test pulse from a $400 \mathrm{~ms},+50 \mathrm{mV}$ conditioning pre-pulse (Fig. 1b). We selected $400 \mathrm{~ms}$ for all pre-pulses because of its physiological relevance to the human cardiac action potential. The gating currents recorded during each test pulse were integrated, and the resultant gating charge was plotted as a function of test potential and fitted with a Boltzmann function (Fig. 1c). Voltage sensor activation displayed a single Boltzmann distribution (Eq. 1, $\mathrm{V}_{1 / 2}:-27.7 \pm 3.8 \mathrm{mV}$ ). In contrast, voltage sensor deactivation surprisingly displayed a double Boltzmann distribution (Eq. 2). Roughly $30 \%$ of the gating charge deactivated with a $V_{1 / 2}$ nearly identical to that measured for VSD activation $(-21.3 \pm 5.3 \mathrm{mV})$, and the remaining $70 \%$ deactivated with a hyperpolarized $\mathrm{V}_{1 / 2}(-52.8 \pm 3.6 \mathrm{mV})$ (Fig. 1C). We also calculated the median voltage of charge transfer 
$\left(V_{\text {Median, }}\right.$ Eq. 3) for each data set (Fig. 1d). $V_{\text {Median }}$ represents the membrane potential where the fully activated and fully resting states are equally populated ${ }^{36}$. This measure is distinct from the $V_{1 / 2}$ because it allows for comparison between Boltzmann distributions that have an unequal number of transitions. The $\mathrm{V}_{\text {Median }}$ of deactivation (-40.4 $\pm 4.5 \mathrm{mV}, n=13$ ), although roughly $10 \mathrm{mV}$ more negative, was not statistically different compared to the $\mathrm{V}_{\text {Median }}$ of activation $(-31.0 \pm 4.2 \mathrm{mV}, n=13, p=0.1)$ (Fig. 1e, Supplemental Table 1). However, comparing the relative charge at each test potential did demonstrate that significantly less charge had moved during VSD deactivation at $-60 \mathrm{mV}$ through $-30 \mathrm{mV}$ compared with VSD activation (Fig. 1c). Taken together, the distinct voltage dependence of VSD activation and deactivation demonstrate that VSD movement is hysteretic at physiological temperature and pulse durations consistent with the human ventricular action potential. These data also suggest that the process of VSD relaxation is intact at elevated temperatures.

Gating current decay during VSD deactivation displays a second time constant that is significantly larger than the single time constant of decay observed during VSD activation, and indicative of the VSD's transition from the relaxed state ${ }^{12}$. We fitted gating current decay with an exponential function (Eq. 4) and observed that at physiological temperature gating currents elicited by depolarization from a $-100 \mathrm{mV}$ holding potential (activation) displayed a single exponential rate of decay. Upon deactivation from +50 $\mathrm{mV}$, the gating currents displayed a double exponential decay (Fig. 1f), consistent with the effects of VSD relaxation. These data provide, to our knowledge, the first characterization of hERG 1 voltage sensor behavior at physiological temperature.

\section{hERG 1a lonic Current Hysteresis at Physiological Temperature}

We then sought to determine how the above reported hERG 1a VSD behavior translated to hERG 1a ionic currents. We recorded the voltage dependence of ionic current activation and deactivation using protocols identical in duration to those used to record gating charge. To assess the voltage dependence of activation we plotted hERG tail currents recorded at $-50 \mathrm{mV}$, as a function of pre-pulse potential and fitted those data with a Boltzmann function (Fig. 2a-c). At room temperature, hERG 1a currents displayed robust hysteresis, as expected, with a $-60.2 \pm 6.8(n=5) \mathrm{mV}$ difference between the voltage dependencies of activation and deactivation (Fig. 2c,d). At physiological temperature hERG activated at more negative potentials and deactivated at more positive potentials compared to room temperature recordings (Fig. 2c, Supplemental Table S2). The convergent shifts in voltage dependence at physiological temperature resulted in a significant reduction in the magnitude of hysteresis at physiological temperature $(-23.6 \pm 1.8$ $\mathrm{mV}, n=12, p<0.0001$ ) compared with room temperature (Fig. 2c,d). Channel kinetics were also accelerated at physiological temperatures compared to room temperature (Fig. 2b,e). The accelerated activation and deactivation kinetics likely drive the concordant shifts in voltage dependence and reduced hysteresis at higher temperatures, as a greater number of channels are able to transition between states during the relatively short $(400 \mathrm{~ms})$ conditioning pulses. These data demonstrate that hysteresis of hERG ionic current is intact, but notably reduced, under physiologically relevant conditions. 


\section{hERG 1a/1b Gating Charge at Physiological Temperature}

Native hERG channels are heteromeric, containing both hERG 1a and hERG $1 \mathrm{~b}$ subunits, and thus have subunits with (hERG 1a) and without (hERG 1b) a functional PAS domain 17,18,37,38. Previous studies completed at room temperature demonstrated that PAS disruption accelerates VSD movement ${ }^{13,15,16}$. These studies suggest that heteromeric hERG 1a/1b channels, comprising PAS-deficient hERG 1b subunits, would display accelerated VSD movement and reduced hysteresis compared to hERG 1a homomeric channels. To test this hypothesis, we repeated the experiments above using a cell line constitutively expressing hERG 1a, but with hERG $1 \mathrm{~b}$ under control of an inducible promoter (see Methods ${ }^{39}$ ) (Fig. 3)

We treated inducible hERG $1 \mathrm{~b}$ cells with $100 \mu \mathrm{g} / \mathrm{ml}$ doxycycline 48 hours prior to recording and validated hERG $1 \mathrm{~b}$ induction by recording the time course of ionic current deactivation (Fig. 3a,b). $100 \mu \mathrm{g} / \mathrm{ml}$ doxycycline significantly accelerated hERG current deactivation, demonstrating that the hERG $1 \mathrm{~b}$ subunit was successfully co-expressed with hERG $1 \mathrm{a}^{39}$. Induced hERG $1 \mathrm{~b}$ expression with doxycycline also depolarized the voltage dependence of deactivation without affecting the voltage dependence of activation (Fig. 3c, Supplemental Table S3), which significantly reduced the magnitude of hysteresis of ionic current (Fig. 3d). These data are consistent with other reports of the impact of hERG $1 \mathrm{~b}$ on hERG 1 channel gating $34,35,40,41$, and demonstrate that $h E R G 1 \mathrm{~b}$ expression was successfully induced.

Inducing hERG $1 \mathrm{~b}$ expression triggered marked and unexpected changes in voltage sensor deactivation, but not activation (Fig. 4). hERG 1b transformed the double Boltzmann distribution generated from hERG 1a alone (Fig. 1c \& 4c) into a single Boltzmann (Fig. 4d), ( $\mathrm{V}_{\text {Median: }}-66.7 \pm 5.3 \mathrm{mV}, p<0.05$, Supplemental Table S4), and increased the hysteresis between VSD activation and deactivation (Fig. 4e). hERG 1b induction did not affect the voltage dependence of activation (Supplemental Table S4). Consequently, the magnitude of hysteresis between voltage sensor activation and deactivation, reported as the difference in $V_{\text {Median, }}$ was significantly larger in cells expressing hERG 1b (Fig. 4e, Supplemental Table S4). The leftward shift of the $V_{\text {Median }}$ of deactivation in heteromeric channels is in opposition with the effects observed on deactivation of ion current, implying that hERG $1 \mathrm{~b}$ alters the communication between the VSD and pore. These data are the first recordings, to our knowledge, from heteromeric $h E R G 1 a / 1 b$ channels. These data also show that hERG $1 \mathrm{~b}$ enhances VSD hysteresis while altering voltage sensor and pore coupling during deactivation.

\section{PAS action determines VSD movement characteristics}

Induced hERG $1 \mathrm{~b}$ expression with hERG 1a hyperpolarized voltage dependence of gating charge deactivation and depolarized the voltage dependence of ionic current deactivation compared to homomeric hERG 1a channels. As hERG $1 \mathrm{~b}$ and hERG 1 a differ only in their N-terminal domains, the reduced hERG 1a PAS domain abundance in heteromeric channels stands out as the most likely culprit underlying the altered VSD behavior in hERG $1 \mathrm{a} / 1 \mathrm{~b}$ channels compared to hERG 1 a channels. 
To determine if the relative activity of the hERG 1a PAS domain is driving the differences in hERG 1a and hERG 1a/1b VSD movement, we characterized the effect of targeted PAS disruption on hERG 1a homomeric channel VSD movement using PAS domain-targeting single chain variable fragment (scFv) antibodies, scFv2.10 and scFv2.12 ${ }^{42}$. scFv2.10 and scF2.12 bind and disrupt PAS action, and thereby induce a heteromeric hERG 1a/1b-like phenotype upon ionic currents recorded from homomeric hERG $1 \mathrm{a}$ channels ${ }^{42,43}$. We therefore hypothesized that PAS disruption with the scFv antibodies would transform hERG 1 a gating currents into a phenotype similar to hERG $1 \mathrm{a} / 1 \mathrm{~b}$ gating currents.

We validated scFv-mediated PAS disruption by recording hERG 1a ionic currents in the presence of each ScFv antibody delivered intracellularly through the recording pipette (Fig. 5). Like hERG 1b, scFv2.10 and scFv2.12 both accelerated the deactivation time course (Fig. $5 c, d, g)$, which we measured by fitting current decay with an exponential function (Eq. 4). The accelerated deactivation time course in the presence of scFv2.10 and scFv2.12 also corresponded with concordant rightward shifts in the voltage dependence of deactivation (Fig. 5e,f, Supplemental Table S1). Despite the mutual shifts in the voltage dependence of deactivation, only scFv2.10 significantly reduced the magnitude of the hysteresis between activation and deactivation (Fig. 5e).

Next, we recorded gating currents at $36 \pm 1^{\circ} \mathrm{C}$ with either scFv2.10 or scFv2.12 (Fig. 6). Similar to the effects of $h E R G 1 b$, both antibodies transformed the double Boltzmann distribution observed under control conditions into a single Boltzmann (Fig. 6b,d), with $\mathrm{V}_{\text {Median }}$ values of $-66.1 \pm 4.4 \mathrm{mV}$ and $-69.0 \pm$ $10.0 \mathrm{mV}$, respectively (Fig. 6e, Supplemental Table S1). Neither antibody affected voltage sensor activation (Fig. 6a,C). Consequently, the hysteresis of gating charge between activation and deactivation was significantly larger in the presence of either PAS-targeting antibody (Fig. 7, Supplemental Table S1). These data demonstrate that PAS action underlies the observed differences in homomeric hERG $1 \mathrm{a}$ and heteromeric hERG1a/1b VSD movement.

\section{Discussion}

Here we sought to measure hERG 1 gating charge at physiological temperatures. In doing so, we identified a fundamental difference in VSD behavior between homomeric hERG 1a and heteromeric hERG $1 \mathrm{a} / 1 \mathrm{~b}$ channels. We found that hERG $1 \mathrm{a}$ voltage sensor movement is hysteretic at near physiological temperature $\left(36^{\circ} \mathrm{C}\right)$. Gating currents elicited by depolarization from a negative holding potential (VSD activation) display a single time constant of decay as well as a Boltzmann distribution. Gating currents elicited by hyperpolarization from a positive potential (VSD deactivation) displayed two time constants of decay and a double Boltzmann distribution. These data demonstrate that VSD relaxation is intact in hERG channels at physiological temperature. Additionally, disrupting PAS function by either induced hERG $1 \mathrm{~b}$ expression or PAS-targeting scFvs transformed the double Boltzmann distribution into a single Boltzmann distribution, and hyperpolarized the voltage dependence of VSD deactivation.

Remarkably, disrupting the PAS domain had an apparent opposing effect at the pore gate, where the voltage dependence of ionic current deactivation was depolarized in the presence of hERG $1 \mathrm{~b}$ or the scFv 
antibodies compared to hERG $1 \mathrm{a}$ alone. The shift is the ionic deactivation could be largely attributed to faster closing and therefore better capture of deactivation at the chosen step duration, instead of an impact on the energetics of pore closure. However, it still stands that the VSD is becoming less likely to return, while the pore is closing faster. The disparate effects on the VSD and pore could suggest that PAS disruption uncouples the voltage sensor from the pore during deactivation.

In this study we provide, to our knowledge, the first report of hERG channel gating charge at physiological temperature. From these recordings there where two key findings. First, VSD movement is hysteretic at physiological temperatures. Delayed hERG pore closure has been suggested to play a cardioprotective role against arrhythmia by prolonging the effective refractory period ${ }^{44,45}$. Our recordings were completed using pulse durations comparable to the human ventricular action potential (400 ms). These data therefore demonstrate that hERG voltage sensor hysteresis, indicative of relaxation, is one mechanism by which pore closure is slowed to enhance hERG channel open probability at the end of the cardiac action potential. Second, VSD deactivation displayed a double Boltzmann distribution, but only from unperturbed hERG 1a homomeric channels. A previous study at room temperature in tsA201 cells reported two charge centers in the hERG VSD that generated a double Boltzmann distribution during VSD activation but not deactivation ${ }^{46}$. The differences in the presentation of the double Boltzmann functions of our study at physiological temperature and the room temperature study could indicate that the charge centers of hERG 1 have distinct $Q_{10}$ values. Two charge systems were also observed in Shakerand $K_{v} 1.5$ channels ${ }^{47,48}$. Our study supports the finding that two charge centers regulate hERG channel gating and expands upon this to demonstrate that the voltage dependencies of these charge centers are distinctly regulated by the PAS domain. We also postulate that the more negative transition $(\approx-53 \mathrm{mV})$ represents recovery from the relaxed state, and the less negative transition $(\approx-21 \mathrm{mV})$ is unrelated to VSD relaxation.

Native cardiac $\mathrm{I}_{\mathrm{Kr}}$ is conducted by a mixed population homomeric and heteromeric $1 \mathrm{a} / 1 \mathrm{~b}$ channels ${ }^{17,18,34}$. hERG $1 \mathrm{~b}$ has a unique, PAS deficient N-terminus, that accelerates the kinetics and enhances the magnitude of ionic current conducted by heteromeric hERG channels compared to homomeric hERG 1 a channels ${ }^{34,40}$. We characterized gating charge recorded from heteromeric channels to generate mechanistic insight into heteromeric channel ionic currents. When we induced hERG $1 \mathrm{~b}$ expression upon hERG 1a expressing cells the resulting heteromeric channel gating currents where nearly identical to currents recorded from scFv-bound hERG 1 a channels. The phenotype of ionic currents recorded from heteromeric $1 \mathrm{a} / 1 \mathrm{~b}$ channels and scFv-bound hERG $1 \mathrm{a}$ channels is also strikingly similar to that observed in channels carrying PAS domain mutations. The most notable of these is R56Q which imparts a heteromeric channel-like gating phenotype without affecting channel trafficking ${ }^{49,50}$. The R56Q mutant accelerates hERG 1a channel gating by disrupting a salt bridge at the interface between the $\mathrm{N}$ terminal PAS and C-terminal CNBHD domains ${ }^{27}$, abolishing the PAS/CNBHD interaction ${ }^{31}$, and effectively disabling the hERG 1a PAS domain. We postulate that ScFv2.10/scFv2.12 binding with the hERG 1a PAS domain disrupts the PAS/CNBHD interaction, effectively disabling the PAS domain as a channel modulator. In vivo, where the relative hERG $1 \mathrm{a}$ and hERG $1 \mathrm{~b}$ levels change developmentally ${ }^{51}$ and 
pathophysiologically ${ }^{52}$, we predict that the stoichiometric relationship of hERG $1 \mathrm{a}$ and hERG $1 \mathrm{~b}$ is used to fine tune $I_{\mathrm{Kr}}$ to meet cardiac demand.

Finally, in two previous studies, scFv2.10 and scFv2.12 were shown to bind the hERG PAS domain and presumably disrupt its ability to regulate channel gating, inducing a PAS-deficient behavior on hERG ionic currents ${ }^{42,43}$. Here we demonstrate that scFv2.10 and scFv2.12, by disrupting PAS action, manipulate hERG VSD movement and its coupling with the pore. Several studies at room temperature have demonstrated that the hERG 1a PAS domain slows VSD movement and enhances gating current hysteresis $^{13,15}$. Those studies also reported concomitant changes in the voltage dependence of ionic currents. More recently, an apparent uncoupling between the hERG VSD and pore gate was reported during prolonged depolarization ${ }^{16}$. In that study, the authors reported that while the voltage dependence of the VSD and pore activation progressively converged with prolonged depolarization, the voltage dependence of VSD and pore deactivation became increasingly disparate ${ }^{16}$. Our study appears to contrast many of these prior findings in that in our hands PAS disruption appeared to simultaneously promote VSD hysteresis and uncouple VSD movement from pore closure during deactivation. We presume that these disparate findings could be consequent to the recording temperatures.

\section{Summary}

The data presented here demonstrate that the hERG $1 \mathrm{a}$ and hERG $1 \mathrm{a} / 1 \mathrm{~b}$ VSD movement is hysteretic at near physiological temperature and time scales. We also identified that PAS action from the hERG $1 \mathrm{a}$ subunit underlies the fundamental differences in VSD deactivation observed in homomeric hERG 1a and heteromeric hERG 1a/1b channels.

\section{Materials Methods}

\section{Cell Culture}

HEK293 cells were cultured in minimum essential medium (MEM, Invitrogen, Cat. No. 11095080) supplemented with $10 \%$ fetal bovine serum (Thermo Fisher, Cat. No. SH30070.03). HEK293 cells stably expressing the hERG 1a subunit alone were cultured in MEM supplemented with $5 \mathrm{mg} / \mathrm{mL}$ of G418 (Thermo Fisher, Cat. No. 11811031). HEK293 cells stably co-expressing hERG 1a and hERG 1b were cultured in MEM medium supplemented with $5 \mathrm{mg} / \mathrm{mL}$ of $\mathrm{G} 418$ and $0.25 \mathrm{mg} / \mathrm{mL}$ of puromycin (Clontech, Cat. 631306). hERG 1b was expressed on demand, using the Tet-One system by Takara (Clontech, Cat. No. 634301). We induced hERG $1 \mathrm{~b}$ protein expression using $100 \mathrm{mg} / \mathrm{ml}$ doxycycline 48 hours prior to recording. Details on the development and maintenance of this cell line were published previously ${ }^{39}$.

\section{Electrophysiology}

All recordings were completed at either room temperature (RT) or near physiological temperature (36 \pm $1^{\circ} \mathrm{C}$ ) using whole-cell patch clamp with an Axon 200A amplifier and Clampex (Molecular Devices). Leak 
subtraction was performed off-line based on measured current observed at potentials negative to hERG 1 activation. The inter-pulse duration for all recordings was 10 seconds.

\section{lonic Recordings}

Data were sampled at $10 \mathrm{kHz}$ and low-pass filtered at $1 \mathrm{kHz}$. Cells were perfused with extracellular solution containing (in $\mathrm{mM}$ ): $150 \mathrm{NaCl}, 5.4 \mathrm{KCl}, 1.8 \mathrm{CaCl}_{2}, 1 \mathrm{MgCl}_{2}, 15$ glucose, $10 \mathrm{HEPES}, 1$ Na-pyruvate, and titrated to $\mathrm{pH} 7.4$ using $\mathrm{NaOH}$. Recording pipettes had resistances of 2-4.5 MW when backfilled with intracellular solution containing (in $\mathrm{mM}$ ): $5 \mathrm{NaCl}, 150 \mathrm{KCl}_{2} 2 \mathrm{CaCl}_{2}, 5$ EGTA, 10 HEPES, $5 \mathrm{MgATP}$ and titrated to $\mathrm{pH} 7.2$ using $\mathrm{KOH}$. We supplemented the intracellular solution with scFv antibodies at $10 \mathrm{mM}$ to measure the effects of the antibodies. Intracellular solution aliquots were kept frozen until the day of recording. The intracellular solution was kept on ice during recordings and discarded 2-3 hours post-thaw.

To assess the voltage dependence of hERG 1 activation, cells were stepped from a $-80 \mathrm{mV}$ holding potential to a 400 millisecond pre-pulse between -100 and $+50 \mathrm{mV}$ in $10 \mathrm{mV}$ increments. Tail currents were then measured during a $-50 \mathrm{mV}, 3$ second test pulse. To assess the voltage dependence of hERG 1 deactivation, cells were stepped from a $-80 \mathrm{mV}$ holding potential to a $+50 \mathrm{mV}$ conditioning pulse for 400 $\mathrm{ms}$ before stepping to a $400 \mathrm{~ms}$ pre-pulse potential between +50 and $-110 \mathrm{mV}$. Tail currents were then measured during a $-50 \mathrm{mV}, 3$ second test pulse. To describe the voltage dependence of activation or deactivation, peak tail current was normalized to cellular capacitance, plotted as a function of pre-pulse potential, and fitted with the following Boltzmann equation:

\section{Equation 1: $\quad y=\left[\frac{A_{1}-A_{2}}{1+e^{\left(V-V_{0}\right) / k}}\right]+A_{2}$}

where $A_{1}$ and $A_{2}$ represent the maximum and minimums of the fit, respectively, $V$ is the membrane potential, $V_{0}$ is the midpoint, and $\mathrm{k}$ is the slope factor.

\section{Gating Currents}

Data were sampled at $20 \mathrm{kHz}$ and low-pass filtered at $10 \mathrm{kHz}$. Cells were perfused with extracellular solution containing (in $\mathrm{mM}$ ): $140 \mathrm{NMDG}, 10 \mathrm{HEPES}, 10$ dextrose, $1 \mathrm{MgCl}_{2}, 1 \mathrm{CaCl}_{2}$, and titrated to $\mathrm{pH} 7.4$ using $\mathrm{HCl}$. Recording pipettes had resistances of 4-7.5 $\mathrm{MW}$ when backfilled with intracellular solution containing (in mM): $140 \mathrm{TEA}-\mathrm{OH}, 1 \mathrm{MgCl}_{2}, 10$ EGTA, and 10 HEPES, adjusted to $\mathrm{pH} 7.2$ using HF. We supplemented the intracellular solution with scFv antibodies at $10 \mathrm{mM}$ to measure the effects of the antibodies. The intracellular solution was kept on ice during recordings.

Following membrane rupture, cells were maintained at -100 $\mathrm{mV}$ for five minutes to allow the pipette solution to fully diffuse into the cell. Capacitance transients were compensated manually at $-120 \mathrm{mV}$ using a $+20 \mathrm{mV}$ test pulse at maximal gain. The capacitance transient was checked after each recording to ensure that it had remained constant. We discarded recordings where the capacitance transient compensation did not remain constant. To assess the voltage dependence of hERG 1 voltage sensor 
activation, cells were stepped from a $-100 \mathrm{mV}$ holding potential to a 400 millisecond pulse between -120 and $+50 \mathrm{mV}$ in $10 \mathrm{mV}$ increments. To assess the voltage dependence of hERG 1 voltage sensor deactivation, cells were stepped from a $-100 \mathrm{mV}$ holding potential to a $+50 \mathrm{mV}$ conditioning pulse for 400 ms before stepping to a $400 \mathrm{~ms}$ pre-pulse potential between +50 and $-120 \mathrm{mV}$.

To describe the voltage dependence of voltage sensor activation or deactivation, the integral of gating currents elicited during each test pulse were plotted as a function of test potential, and fitted with either a single Boltzmann equation ( $E q .1$ ) or a double Boltzmann equation:

Equation 2: $\quad y=\left[\left(\frac{A_{1}}{1+e^{\left(V-V_{1}\right) / k_{1}}}\right)+\left(\frac{A_{2}}{1+e^{\left(V-V_{2}\right) / k_{2}}}\right)\right]+A_{3}$,

where $A_{1}$ is the relative amplitude of transition $1, A_{2}$ is the relative amplitude of transition $2, A_{3}$ is the minimum of the fit, $V$ is the membrane potential, $V_{1}$ and $V_{2}$ are the respective midpoints of each transition, and $k_{1}$ and $k_{2}$ are the respective slope factors of each transition. We derived the $V_{\text {Median }}$ for each charge versus voltage $(\mathrm{QV})$ curve by measuring the area between the curve and the ordinate $(\mathrm{Q})$ axis as described by Chowdhury and Chanda ${ }^{36}$, where the area between the curve and the $Q$ axis is calculated using the trapezoid method:

\section{Equation 3: $\quad V_{\text {Median }}=\frac{1}{2} \sum_{i=1}^{n-1}\left(V_{i+1}+V_{i}\right)\left(Q_{i+1}-Q_{i}\right)$,}

where $V_{i}$ is the $i^{\text {th }}$ point on the QV curve and $Q_{i}$ is the fraction of charge that is transferred at $V_{i}$.

lonic and gating current kinetics were assessed by fitting current decay during the test pulse with a single or a double exponential function:

\section{Equation 4: $\quad y=Y_{0}+A_{1} e^{-t / \tau_{1}}+A_{2} e^{-t / \tau_{2}}$,}

where $Y_{0}$ is the asymptote, $A_{1}$ and $A_{2}$ are the relative components of the fast and slow time constants $t_{1}$ and $t_{2}$, respectively.

\section{Statistical Analysis}

Analysis was completed using Clampfit (Molecular Devices) and Origin (OriginLab). All data are reported as mean \pm SEM and were compared using a Student's t-tests. When applicable, an ANOVA and Bonferroni post hoct-tests where used. Statistical significance was taken at $p<0.05$. Data points greater than two times the standard deviation were termed outliers and excluded from analysis.

\section{Data Availability}


The datasets generated during and/or analyzed during the current study are available from the corresponding author on reasonable request.

\section{Declarations}

\section{Acknowledgements}

The author thanks Dr. Gail Robertson and Dr. Tom Claydon for critical manuscript feedback. The scFv antibodies were kindly provided by Dr. Carol Harley and Dr. Joao Morais Cabral. This work was supported by NIH/NHLBI R00HL133482 (to D.K. Jones) and NIH/NINDS 5R01 NS081320 (to G. A. Robertson.

\section{Author Contributions}

D. K. J. conceived and conducted the experiments, analyzed the results, and reviewed the manuscript.

\section{Author Contributions}

The author declares no competing interests.

\section{References}

1. Trudeau, M. C., Warmke, J. W., Ganetzky, B. \& Robertson, G. A. HERG, a human inward rectifier in the voltage-gated potassium channel family. Science 269, 92-95 (1995).

2. Sanguinetti, M. C., Jiang, C., Curran, M. E. \& Keating, M. T. A mechanistic link between an inherited and an acquired cardiac arrhythmia: HERG encodes the IKr potassium channel. Cel/ 81, 299-307 (1995).

3. Curran, M. E. et al. A molecular basis for cardiac arrhythmia: HERG mutations cause long QT syndrome. Cel/ 80, 795-803 (1995).

4. Wang, W. \& MacKinnon, R. Cryo-EM Structure of the Open Human Ether-a-go-go-Related K(+) Channel hERG. Cell 169, 422-430 e410, doi:10.1016/j.cell.2017.03.048 (2017).

5. Long, S. B., Campbell, E. B. \& Mackinnon, R. Crystal structure of a mammalian voltage-dependent Shaker family K+ channel. Science 309, 897-903 (2005).

6. Hodgkin, A. L. \& Huxley, A. F. A quantitative description of membrane current and its application to conduction and excitation in nerve. J Physio/ 117, 500-544 (1952).

7. Armstrong, C. M. \& Bezanilla, F. Currents related to movement of the gating particles of the sodium channels. Nature 242, 459-461 (1973).

8. Bezanilla, F., White, M. M. \& Taylor, R. E. Gating currents associated with potassium channel activation. Nature 296, 657-659 (1982).

9. Piper, D. R., Sanguinetti, M. C. \& Tristani-Firouzi, M. Voltage sensor movement in the hERG K+ channel. Novartis Found Symp 266, 46-52; discussion 52-46, 95-49 (2005). 
10. Goodchild, S. J. \& Fedida, D. Gating charge movement precedes ionic current activation in hERG channels. Channels (Austin) 8, 84-89, doi:10.4161/chan.26775 (2014).

11. Dou, Y., Macdonald, L. C., Wu, Y. \& Fedida, D. The Fast Component of hERG Gating Charge: An Interaction between D411 in the S1 and S4 Residues. Biophys J 113, 1979-1991, doi:10.1016/j.bpj.2017.09.004 (2017).

12. Villalba-Galea, C. A., Sandtner, W., Starace, D. M. \& Bezanilla, F. S4-based voltage sensors have three major conformations. Proc Natl Acad Sci U S A 105, 17600-17607, doi:10.1073/pnas.0807387105 (2008).

13. Goodchild, S. J., Macdonald, L. C. \& Fedida, D. Sequence of gating charge movement and pore gating in HERG activation and deactivation pathways. Biophys $\mathrm{J} 108,1435-1447$, doi:10.1016/j.bpj.2015.02.014 (2015).

14. Bezanilla, F., Taylor, R. E. \& Fernandez, J. M. Distribution and kinetics of membrane dielectric polarization. 1. Long-term inactivation of gating currents. Journal of General Physiology 79, 21-40 (1982).

15. Tan, P. S., Perry, M. D., Ng, C. A., Vandenberg, J. I. \& Hill, A. P. Voltage-sensing domain mode shift is coupled to the activation gate by the N-terminal tail of hERG channels. J Gen Physiol 140, 293-306, doi:10.1085/jgp.201110761 (2012).

16. Thouta, S. et al. Stabilization of the Activated hERG Channel Voltage Sensor by Depolarization Involves the S4-S5 Linker. Biophys J 112, 300-312, doi:10.1016/j.bpj.2016.12.021 (2017).

17. Jones, D. K. et al. hERG $1 \mathrm{~b}$ is critical for human cardiac repolarization. Proc Natl Acad Sci U S A 111, 18073-18077, doi:10.1073/pnas.1414945111 (2014).

18. Jones, E. M., Roti Roti, E. C., Wang, J., Delfosse, S. A. \& Robertson, G. A. Cardiac IKr channels minimally comprise hERG 1a and 1b subunits. J Biol Chem 279, 44690-44694 (2004).

19. London, B. et al. Two isoforms of the mouse ether-a-go-go-related gene coassemble to form channels with properties similar to the rapidly activating component of the cardiac delayed rectifier K+ current. Circ Res 81, 870-878 (1997).

20. Lees-Miller, J. P., Kondo, C., Wang, L. \& Duff, H. J. Electrophysiological characterization of an alternatively processed ERG K+ channel in mouse and human hearts. Circ Res 81, 719-726 (1997).

21. Goversen, B. et al. The influence of hERG1a and hERG1b isoforms on drug safety screening in iPSCCMs. Prog Biophys Mol Biol, doi:10.1016/j.pbiomolbio.2019.02.003 (2019).

22. Morais Cabral, J. H. et al. Crystal structure and functional analysis of the HERG potassium channel $\mathrm{N}$ terminus: a eukaryotic PAS domain. Cell 95, 649-655 (1998).

23. Gianulis, E. C., Liu, Q. \& Trudeau, M. C. Direct interaction of eag domains and cyclic nucleotidebinding homology domains regulate deactivation gating in hERG channels. J Gen Physio/ 142, 351366, doi:10.1085/jgp.201310995 (2013).

24. Hull, C. M., Sokolov, S., Van Slyke, A. C. \& Claydon, T. W. Regional flexibility in the S4-S5 linker regulates hERG channel closed-state stabilization. Pflugers Arch 466, 1911-1919, doi:10.1007/s00424-013-1431-9 (2014). 
25. Wang, J., Myers, C. D. \& Robertson, G. A. Dynamic control of deactivation gating by a soluble aminoterminal domain in HERG K(+) channels. J Gen Physiol 115, 749-758 (2000).

26. Wang, J., Trudeau, M. C., Zappia, A. M. \& Robertson, G. A. Regulation of deactivation by an amino terminal domain in human ether-a- go-go-related gene potassium channels [published erratum appears in J Gen Physiol 1999 Feb;113(2):359]. J Gen Physiol 112, 637-647 (1998).

27. Ng, C. A., Phan, K., Hill, A. P., Vandenberg, J. I. \& Perry, M. D. Multiple interactions between cytoplasmic domains regulate slow deactivation of Kv11.1 channels. J Biol Chem 289, 2582225832, doi:10.1074/jbc.M114.558379 (2014).

28. Haitin, Y., Carlson, A. E. \& Zagotta, W. N. The structural mechanism of KCNH-channel regulation by the eag domain. Nature 501, 444-448, doi:10.1038/nature12487 (2013).

29. Gustina, A. S. \& Trudeau, M. C. The eag domain regulates $h E R G$ channel inactivation gating via a direct interaction. J Gen Physio/ 141, 229-241, doi:10.1085/jgp.201210870 (2013).

30. Gustina, A. S. \& Trudeau, M. C. A recombinant N-terminal domain fully restores deactivation gating in $\mathrm{N}$-truncated and long QT syndrome mutant hERG potassium channels. Proc Natl Acad Sci U S A 106, 13082-13087, doi:10.1073/pnas.0900180106 (2009).

31. Gianulis, E. C. \& Trudeau, M. C. Rescue of aberrant gating by a genetically encoded PAS (Per-ArntSim) domain in several long QT syndrome mutant human ether-a-go-go-related gene potassium channels. J Biol Chem 286, 22160-22169, doi:10.1074/jbc.M110.205948 (2011).

32. Ng, C. A. et al. C-Terminal beta9-Strand of the Cyclic Nucleotide-Binding Homology Domain Stabilizes Activated States of Kv11.1 Channels. PloS one 8, e77032, doi:10.1371/journal.pone.0077032 (2013).

33. Liu, Q. N. \& Trudeau, M. C. Eag Domains Regulate LQT Mutant hERG Channels in Human Induced Pluripotent Stem Cell-Derived Cardiomyocytes. PloS one 10, e0123951, doi:10.1371/journal.pone.0123951 (2015).

34. Sale, H. et al. Physiological properties of hERG 1a/1b heteromeric currents and a hERG $1 \mathrm{~b}$-specific mutation associated with Long-QT syndrome. Circ Res 103, e81-95, doi:10.1161/CIRCRESAHA.108.185249 (2008).

35. Trudeau, M. C., Leung, L. M., Roti, E. R. \& Robertson, G. A. hERG1a N-terminal eag domain-containing polypeptides regulate homomeric $h E R G 1 b$ and heteromeric $h E R G 1 a / h E R G 1 b$ channels: a possible mechanism for long QT syndrome. J Gen Physio/ 138, 581-592, doi:10.1085/jgp.201110683 (2011).

36. Chowdhury, S. \& Chanda, B. Estimating the voltage-dependent free energy change of ion channels using the median voltage for activation. J Gen Physio/ 139, 3-17, doi:10.1085/jgp.201110722 (2012).

37. Jones, D. K., Liu, F., Dombrowski, N., Joshi, S. \& Robertson, G. A. Dominant negative consequences of a hERG $1 \mathrm{~b}$-specific mutation associated with intrauterine fetal death. Prog Biophys Mol Biol 120, 6776, doi:10.1016/j.pbiomolbio.2016.01.002 (2016).

38. London, B. et al. N and C-terminal isoforms of HERG in the human heart. Biophysical J. (1998).

39. Rios-Perez, E. B. et al. A stable cell line inducibly expressing hERG1a/1b heteromeric channels. J Pharmacol Toxicol Methods 110, 107081, doi:10.1016/j.vascn.2021.107081 (2021). 
40. McPate, M. J. et al. hERG1a/1b heteromeric currents exhibit amplified attenuation of inactivation in variant 1 short QT syndrome. Biochem Biophys Res Commun 386, 111-117, doi:10.1016/j.bbrc.2009.05.134 (2009).

41. Larsen, A. P., Olesen, S.P., Grunnet, M., Jespersen, T.. Characterization of hERG1a and hERG1b potassium channels - a possible role for hERG1b in the IKr current. Pflugers Arch DOI 10.1007/s00424-008-0476-7 (2008).

42. Harley, C. A. et al. Enhancement of hERG channel activity by scFv antibody fragments targeted to the PAS domain. Proc Natl Acad Sci U S A 113, 9916-9921, doi:10.1073/pnas.1601116113 (2016).

43. Harley, C. A. et al. Conformation-sensitive antibody reveals an altered cytosolic PAS/CNBh assembly during hERG channel gating. Proc Natl Acad Sci U S A 118, doi:10.1073/pnas.2108796118 (2021).

44. Shi, Y. P. et al. The hERG channel activator, RPR260243, enhances protective IKr current in early refractory reducing arrhythmogenicity in zebrafish hearts. Am J Physiol Heart Circ Physiol, doi:10.1152/ajpheart.00038.2020 (2020).

45. Lu, Y. et al. Effects of premature stimulation on HERG K(+) channels. J Physio/ 537, 843-851, doi:10.1111/j.1469-7793.2001.00843.x (2001).

46. Wang, Z., Dou, Y., Goodchild, S. J., Es-Salah-Lamoureux, Z. \& Fedida, D. Components of gating charge movement and S4 voltage-sensor exposure during activation of hERG channels. J Gen Physio/ 141, 431-443, doi:10.1085/jgp.201210942 (2013).

47. Bezanilla, F., Perozo, E. \& Stefani, E. Gating of Shaker K+ channels: II. The components of gating currents and a model of channel activation. Biophys J 66, 1011-1021 (1994).

48. Hesketh, J. C. \& Fedida, D. Sequential gating in the human heart $\mathrm{K}(+)$ channel Kv1.5 incorporates $\mathrm{Q}(1)$ and Q(2) charge components. The American journal of physiology 277, H1956-1966, doi:10.1152/ajpheart.1999.277.5.H1956 (1999).

49. Harley, C. A., Jesus, C. S., Carvalho, R., Brito, R. M. \& Morais-Cabral, J. H. Changes in channel trafficking and protein stability caused by LQT2 mutations in the PAS domain of the HERG channel. PloS one 7, e32654, doi:10.1371/journal.pone.0032654 (2012).

50. Chen, J., Zou, A., Splawski, I., Keating, M. T. \& Sanguinetti, M. C. Long QT syndrome-associated mutations in the Per-Arnt-Sim (PAS) domain of HERG potassium channels accelerate channel deactivation. J Biol Chem 274, 10113-10118 (1999).

51. Crotti, L. et al. Long QT syndrome-associated mutations in intrauterine fetal death. Jama 309, 14731482, doi:10.1001/jama.2013.3219 (2013).

52. Holzem, K. M. et al. Reduced response to I blockade and altered hERG1a/1b stoichiometry in human heart failure. J Mol Cell Cardiol, doi:10.1016/j.yjmcc.2015.06.008 (2015).

\section{Figures}


a

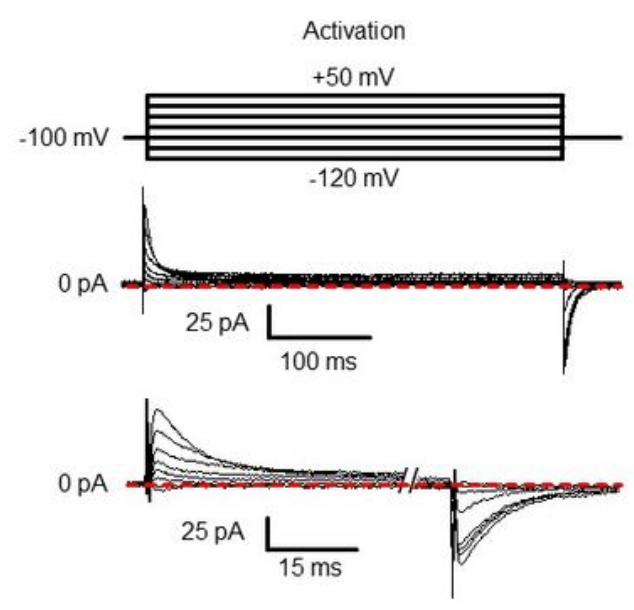

C

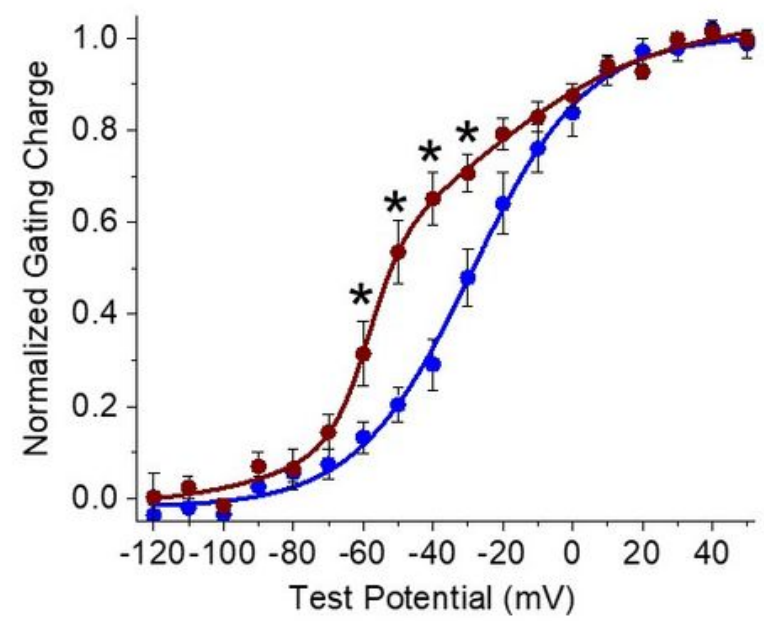

e

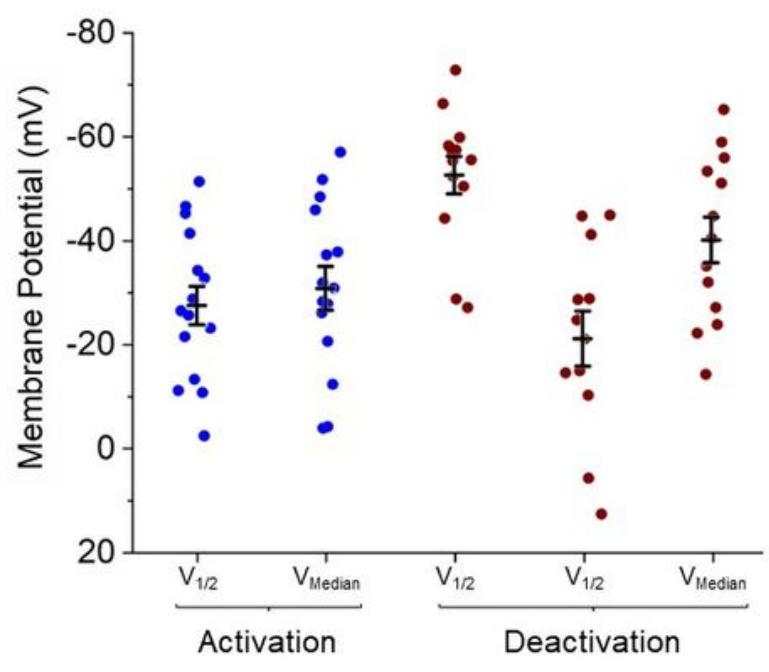

b

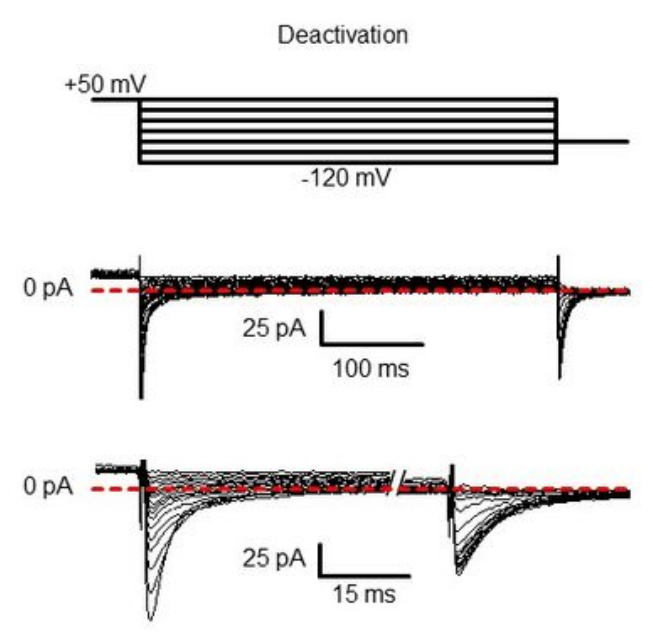

d

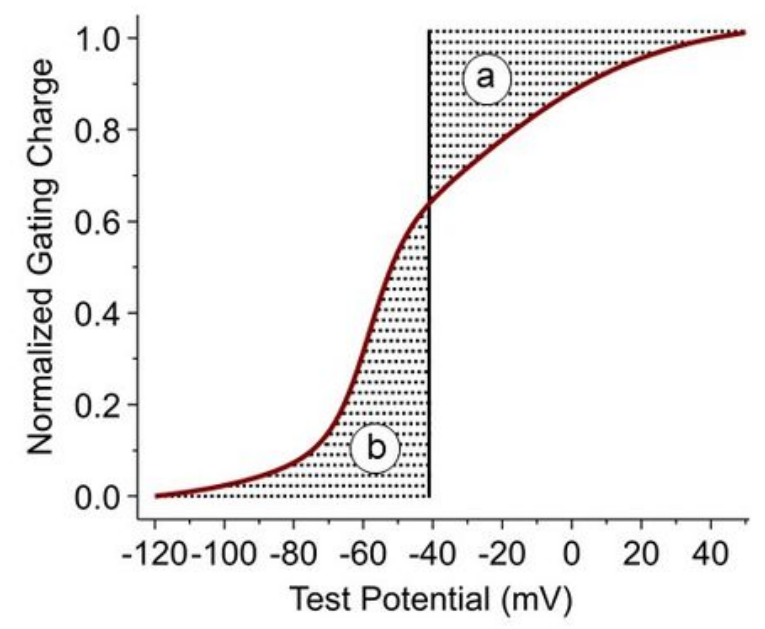

f

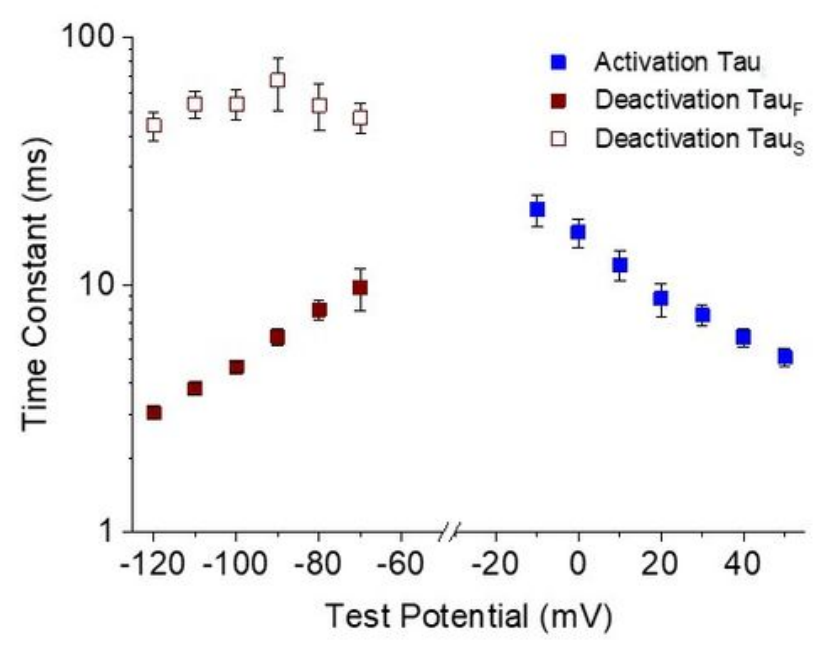

\section{Figure 1}

Hysteretic Behavior of hERG 1a gating charge at near physiological temperature $\left(36^{\circ} \mathrm{C}\right)$. (a) The pulse protocol (top) and corresponding activation gating current traces (middle). An expanded view of the current traces is shown at bottom. hERG 1a gating charge activation was measured by recording gating currents elicited by depolarization from a negative $(-100 \mathrm{mV})$ holding potential. (b) The pulse protocol (top) and corresponding deactivation gating current traces (middle). An expanded view of the current 
traces is shown at bottom. hERG 1a gating charge deactivation was measured by recording gating currents elicited by hyperpolarization from a positive $(+50 \mathrm{mV})$ holding potential. (c) Voltage dependence of hERG 1a voltage sensor activation (b/ue) and deactivation (red). Gating currents elicited during the test pulse were integrated, and the resultant gating charge was normalized, plotted as a function of test potential and fitted with either a single Boltzmann (activation) or a double Boltzmann (deactivation) function. The majority of gating charge deactivation occurred at membrane potentials significantly more negative than activation. (d) Graphical representation of the median voltage of charge movement $\left(V_{\text {median }}\right)$. The shaded areas " $a$ " and " $b$ " represent the area between the curve and the ordinate axis. The $V_{\text {median }}$ is the point along the $X$ axis where the absolute value of $a$ and $b$ are equal. (e) Bar graph depicting mean $V_{1 / 2}$ values and $V_{\text {median }}$ values for VSD activation and deactivation. (f) Time constants of gating current decay are hysteretic. We fitted the decay of gating currents elicited during each test pulse with a single (activation, blue) or double exponential function (deactivation, red). All data are reported as mean \pm SEM. * indicates $p<0.05 . n=12$ 
a

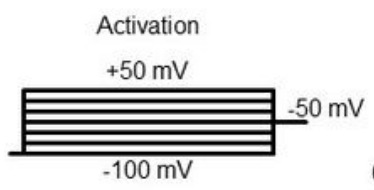

b

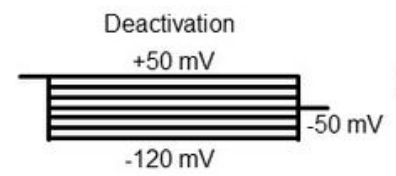

C

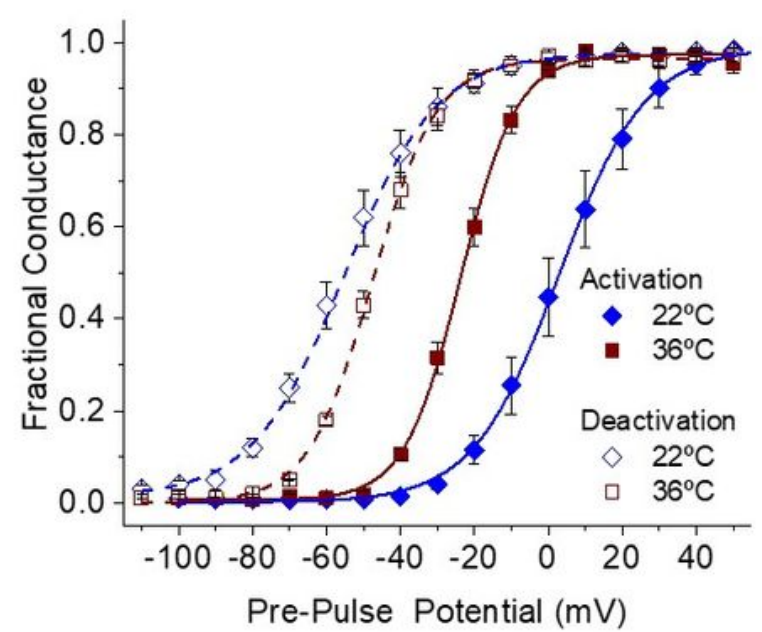

$22^{\circ} \mathrm{C}$
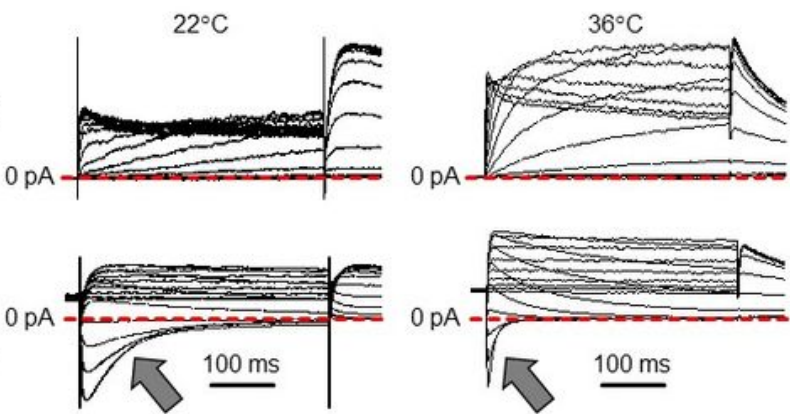

d

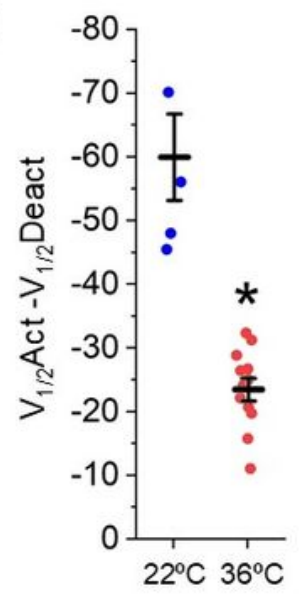

e

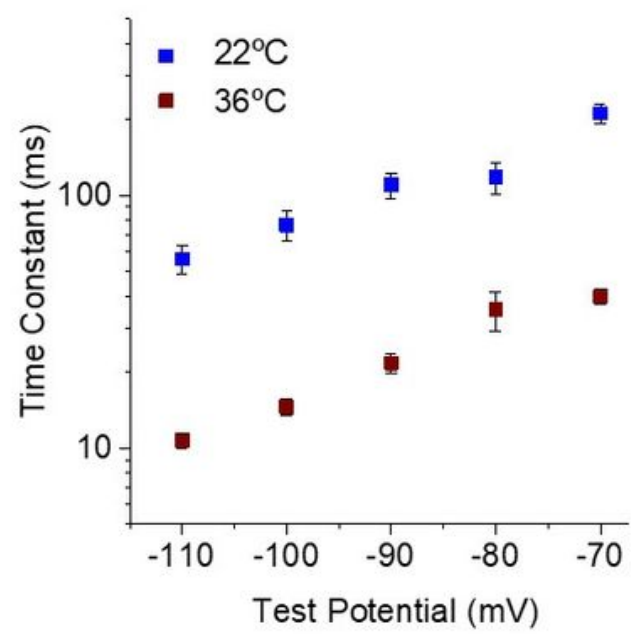

Figure 2

Physiological temperature reduces hERG 1a hysteresis of ionic currents. (a) Pulse protocol and corresponding sample ionic traces showing hERG 1 a activation when stably expressed in HEK293 cells recorded at room temperature $\left(22^{\circ} \mathrm{C}\right.$, middle) or physiological temperature $\left(36^{\circ} \mathrm{C}\right.$, right). (b) Pulse protocol and corresponding sample ionic traces showing hERG 1 a deactivation when stably expressed in HEK293 cells recorded at room temperature $\left(22^{\circ} \mathrm{C}\right.$, middle) or physiological temperature $\left(36^{\circ} \mathrm{C}\right.$, right $)$. (c) 
Temperature-dependent shift in hERG 1a voltage dependence. Normalized peak tail current, representing fractional conductance, is plotted as a function of pre-pulse potential and fitted with a Boltzmann function for recordings completed at $22^{\circ} \mathrm{C}$ (blue diamonds) and $36^{\circ} \mathrm{C}$ (red squares). Channel activation recorded as in "a" is shown with solid symbols. Channel deactivation recorded as in " $b$ " is shown with open symbols. (d) The magnitude of hysteresis was quantified by subtracting the $V_{1 / 2}$ of deactivation from the $\mathrm{V}_{1 / 2}$ of activation. Hysteresis was significantly smaller in recordings completed at $36^{\circ} \mathrm{C}(n=12)$ compared with recordings at $22^{\circ} \mathrm{C}(n=5)$. (e) Time constants representing the fast deactivation time course for hERG 1 a current recordings completed at either $22^{\circ} \mathrm{C}$ (b/ue) or $36^{\circ} \mathrm{C}$ (red). We fit current decay (arrows in "b") with a double exponential equation (eq. 4) and reported the fast time constant (t) as a function of test potential. All data are reported as mean \pm SEM. * indicates $p<0.05$. 
a
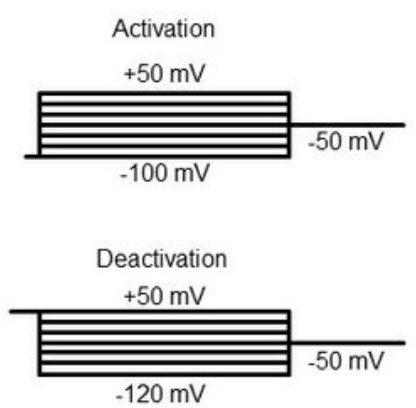

hERG 1a
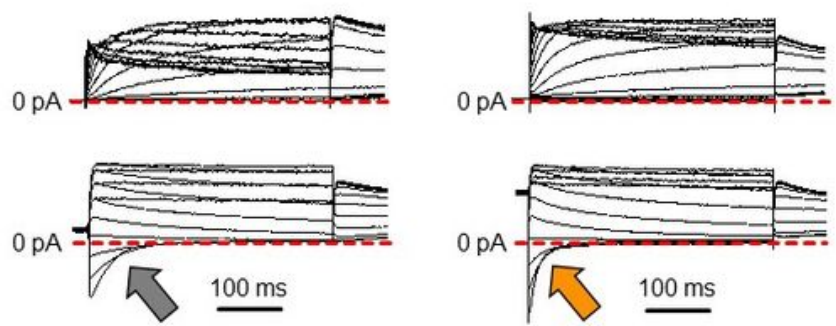

b

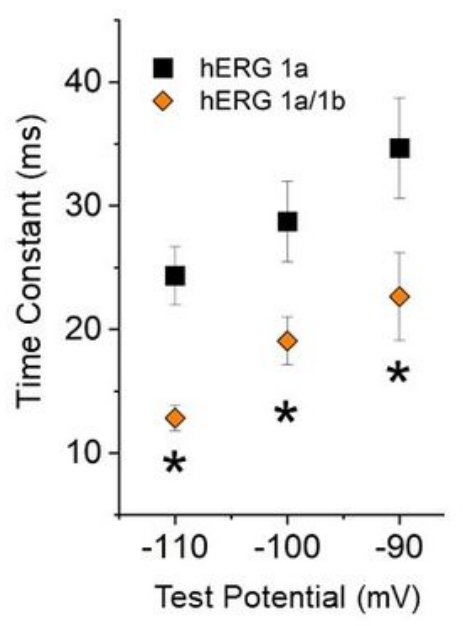

c

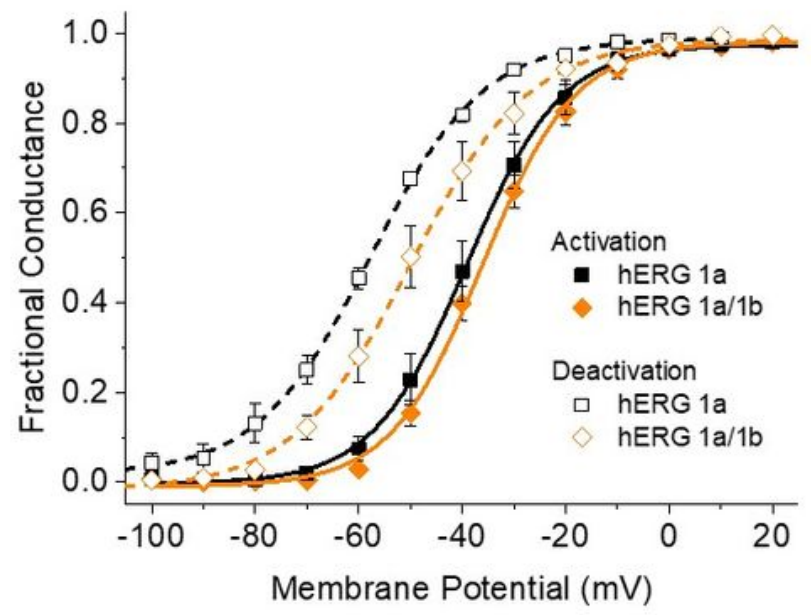

d

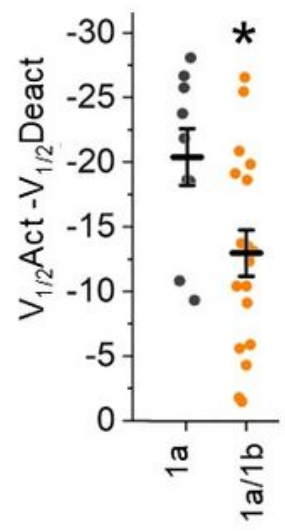

\section{Figure 3}

hERG 1b expression reduces hERG channel hysteresis of ionic current. (a) Pulse protocol and corresponding sample ionic traces showing hERG 1a (middle) and hERG 1a/1b channel (right) activation when stably expressed in HEK293 cells recorded at physiological temperature $\left(36^{\circ} \mathrm{C}\right)$. (b) Pulse protocol and corresponding sample ionic traces showing hERG 1a (middle) and hERG 1a/1b channel (right) deactivation when stably expressed in HEK293 cells recorded at physiological temperature $\left(36^{\circ} \mathrm{C}\right)$. (c) 
hERG 1b modifies hERG channel voltage dependence. Normalized peak tail current, representing fractional conductance, is plotted as a function of pre-pulse potential and fitted with a Boltzmann function for hERG 1a (black) and hERG 1a/1b (orange). Channel activation recorded as in " $a$ " is shown with solid symbols. Channel deactivation recorded as in " $B$ " is shown with open symbols. (d) The magnitude of hysteresis was quantified by subtracting the $V_{1 / 2}$ of deactivation from the $V_{1 / 2}$ of activation. Hysteresis was significantly smaller in hERG 1a/1b heteromeric channels $(n=10)$ compared with hERG 1a homomeric channels $(n=7)$. All data are reported as mean \pm SEM. * indicates $p<0.05$.

a

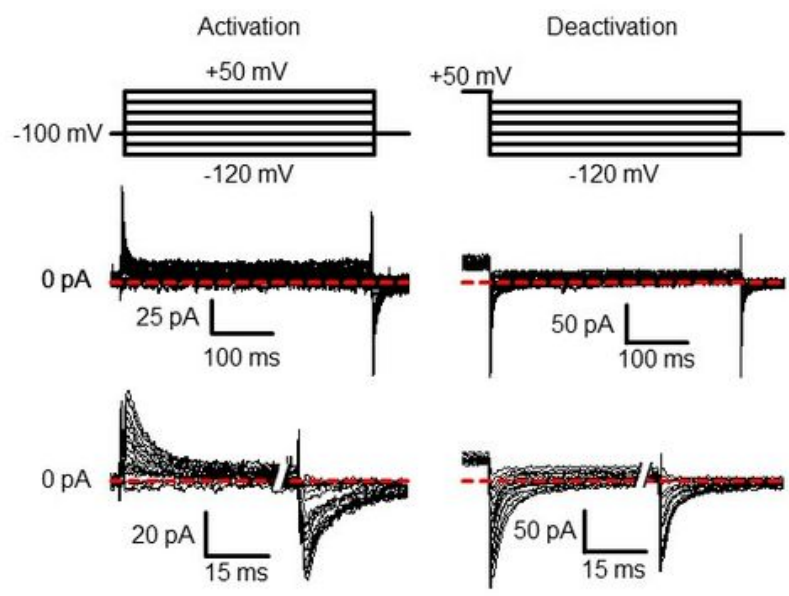

b

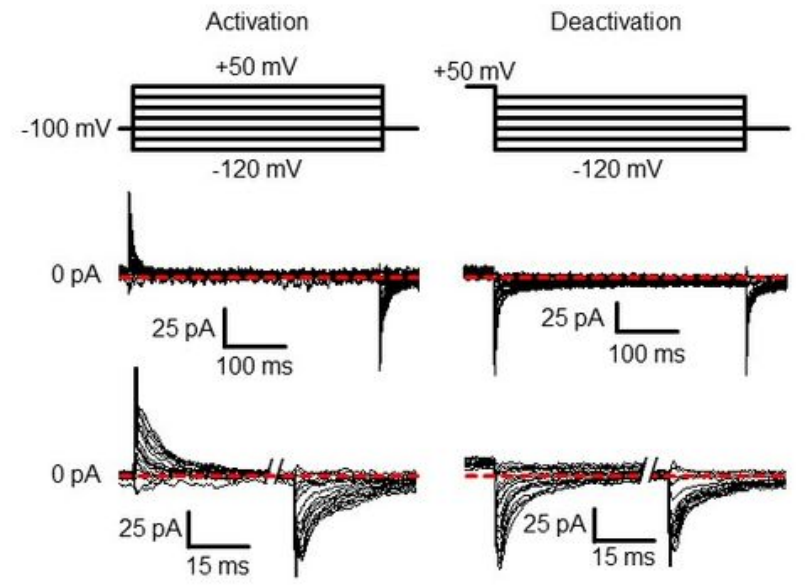

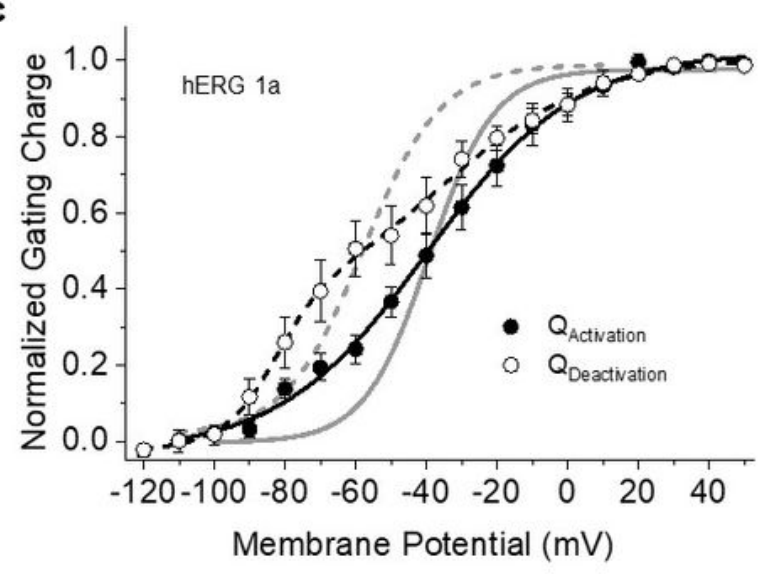

d

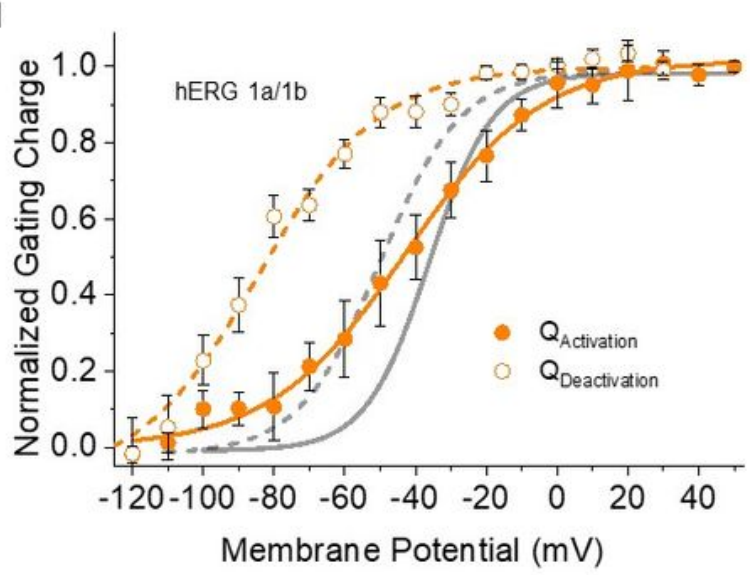

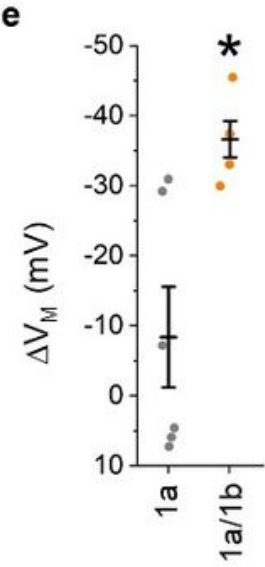




\section{Figure 4}

hERG 1b expression transforms hERG 1a channel gating. hERG 1b expression transforms hERG channel gating charge. (a) Activation and deactivation pulse protocols (top) and corresponding activation and deactivation gating current traces recorded at $36 \pm 1^{\circ} \mathrm{C}$ from HEK293 cells stably expressing hERG $1 \mathrm{a}$. An expanded view of the gating current traces is show at bottom. (b) Activation and deactivation pulse protocols (top) and corresponding activation and deactivation gating current traces recorded at $36 \pm 1{ }^{\circ} \mathrm{C}$ from HEK293 cells stably expressing hERG 1a and hERG 1b. An expanded view of the gating current traces is show at bottom. (c) Voltage dependence of hERG 1a gating charge activation (filled circles) and deactivation (open circles). Gating currents elicited during the test pulse were integrated, and the resultant gating charge was normalized, plotted as a function of test potential and fitted with a Boltzmann function. Voltage dependence of ionic current activation and deactivation are shown in gray. (d) Voltage dependence of hERG 1a/1b gating charge activation (filled circles) and deactivation (open circles). Voltage dependence of ionic current activation and deactivation are shown in gray. Expression of hERG $1 \mathrm{~b}$ with hERG 1a triggered disparate shifts in the voltage dependence of VSD and ionic deactivation. (e) The magnitude of hysteresis was quantified by subtracting the $V_{\text {median }}$ of deactivation from the $V_{\text {median }}$ of activation. Hysteresis was significantly larger when hERG $1 \mathrm{~b}$ was coexpressed with hERG 1a $(n=10)$ compared with hERG 1a expressed alone $(n=8)$. All data are reported as mean \pm SEM. * indicates $p<$ 0.05 . 

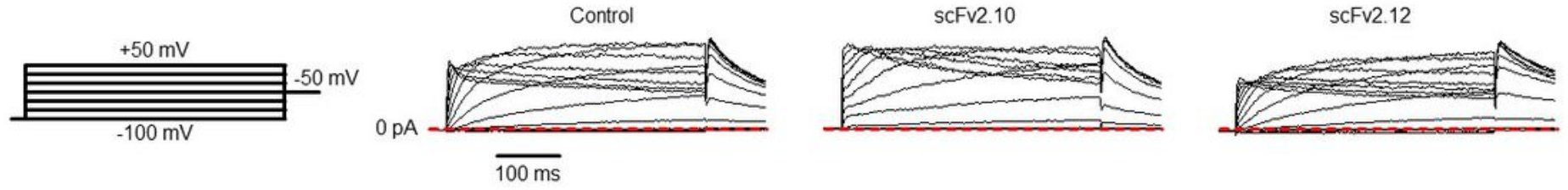

b
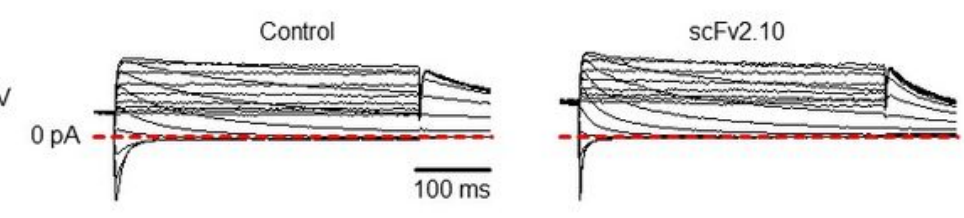

scFv2.12
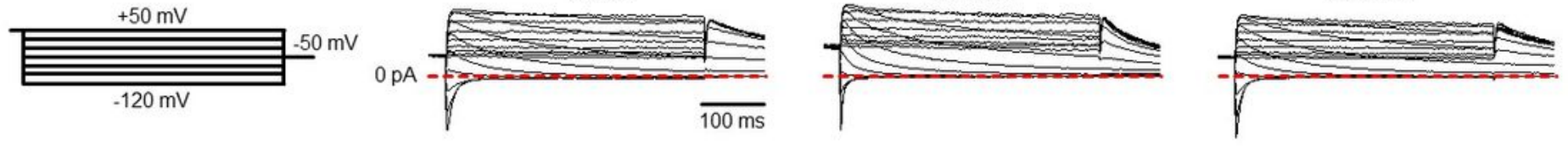

C
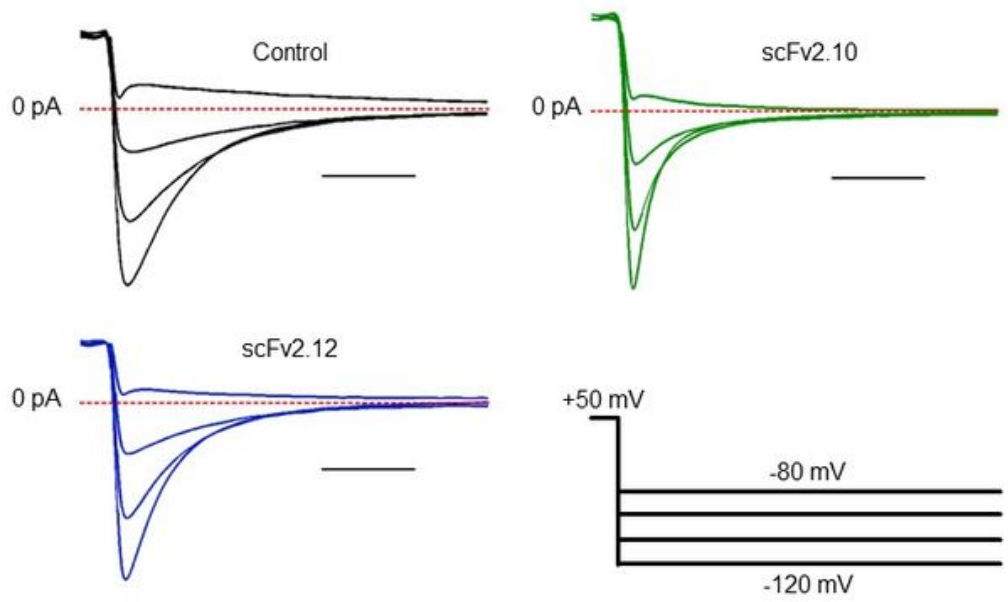

e
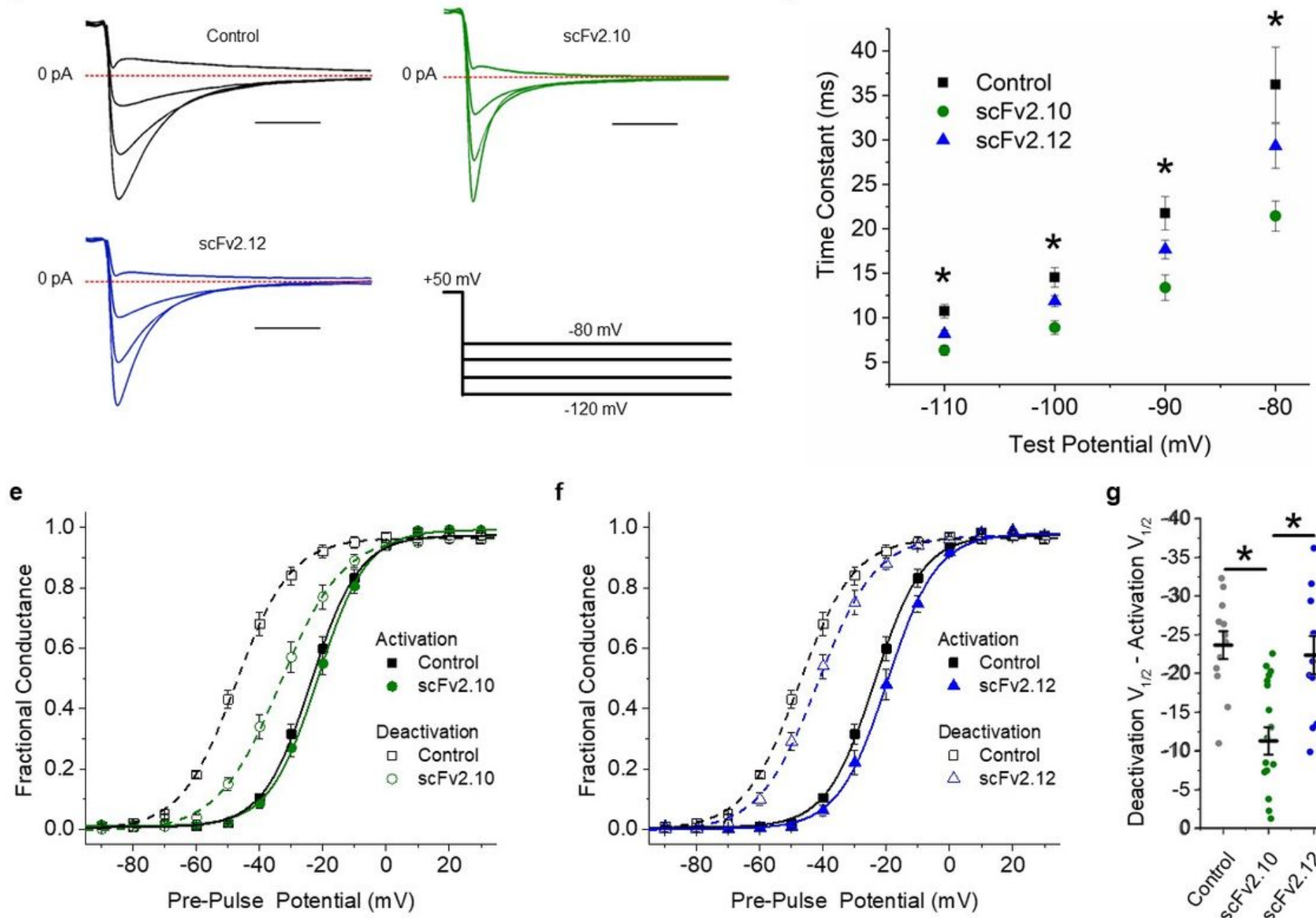

f
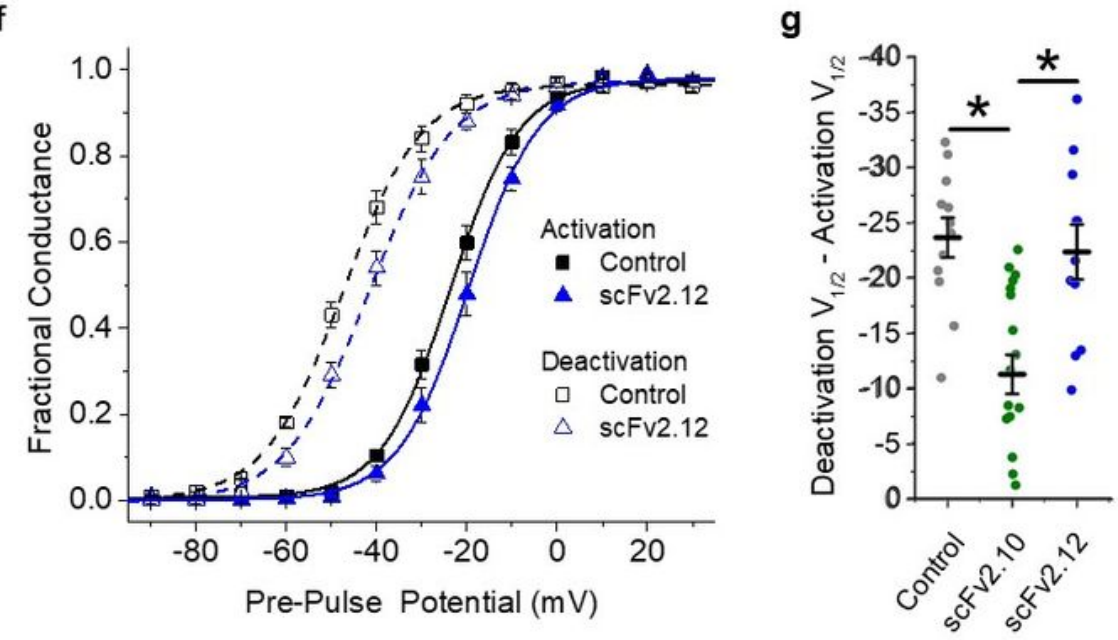

\section{Figure 5}

Effects of anti-PAS antibodies on hERG 1a ionic current hysteresis. (a) Pulse protocol (left) and corresponding sample ionic traces showing hERG 1a activation in vehicle control solution or in the presence of $10 \mu \mathrm{M}$ scFv2.10 or $10 \mu \mathrm{M}$ scFv2.12. (b) Pulse protocol (left) and corresponding sample ionic traces showing hERG 1 a deactivation in vehicle control solution or in the presence of scFv2.10 or scFv2.12. (c) scFv2.10 modification of hERG 1a voltage dependence. Normalized peak tail current, 
representing fractional conductance, is plotted as a function of pre-pulse potential and fitted with a Boltzmann function for hERG 1a recordings in vehicle control solution (black) and scFv2.10 (green). (d) scFv2.12 modification of hERG 1a voltage dependence. Normalized peak tail current, representing fractional conductance, is plotted as a function of pre-pulse potential and fitted with a Boltzmann function for hERG 1a recordings in vehicle control solution (black) and scFv2.12 (b/ue). (e) The magnitude of hysteresis was quantified by subtracting the $V_{1 / 2}$ of deactivation from the $V_{1 / 2}$ of activation. Hysteresis was significantly smaller in the presence of scFv2.10 $(n=14)$ compared with control recordings $(n=12)$ and recordings in the presence of scFv2.12 $(n=6)$. (f) Expanded view of deactivating hERG 1a ionic currents recorded from Control (black), scFv2.10 (green) and scFv2.12 (b/ue) cells. (g) Fast time constants of deactivation plotted as a function of test potential for Control (black), scFv2.10 (green) and scFv2.12 (b/ue) cells. Time constants were derived by fitting current decay with a double exponential function. All data are reported as mean \pm SEM. * indicates $p<0.05$. 
a
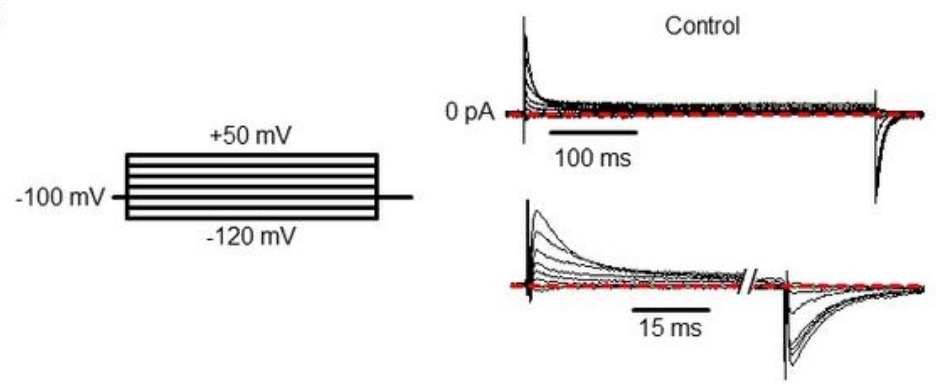

b

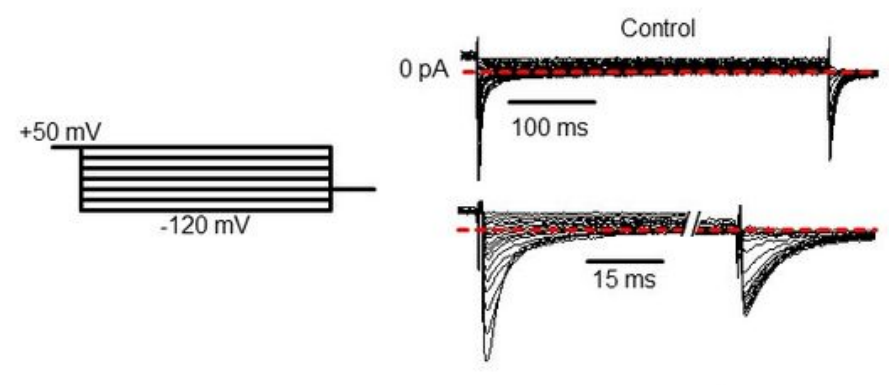

c

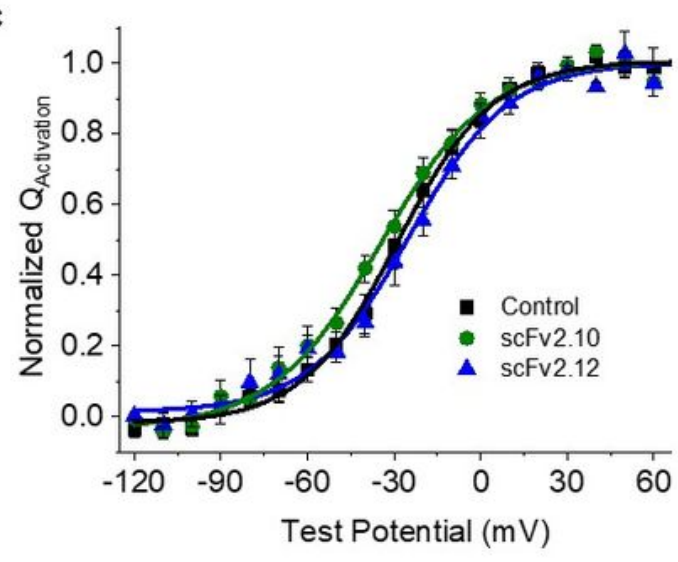

d
scFv2.10
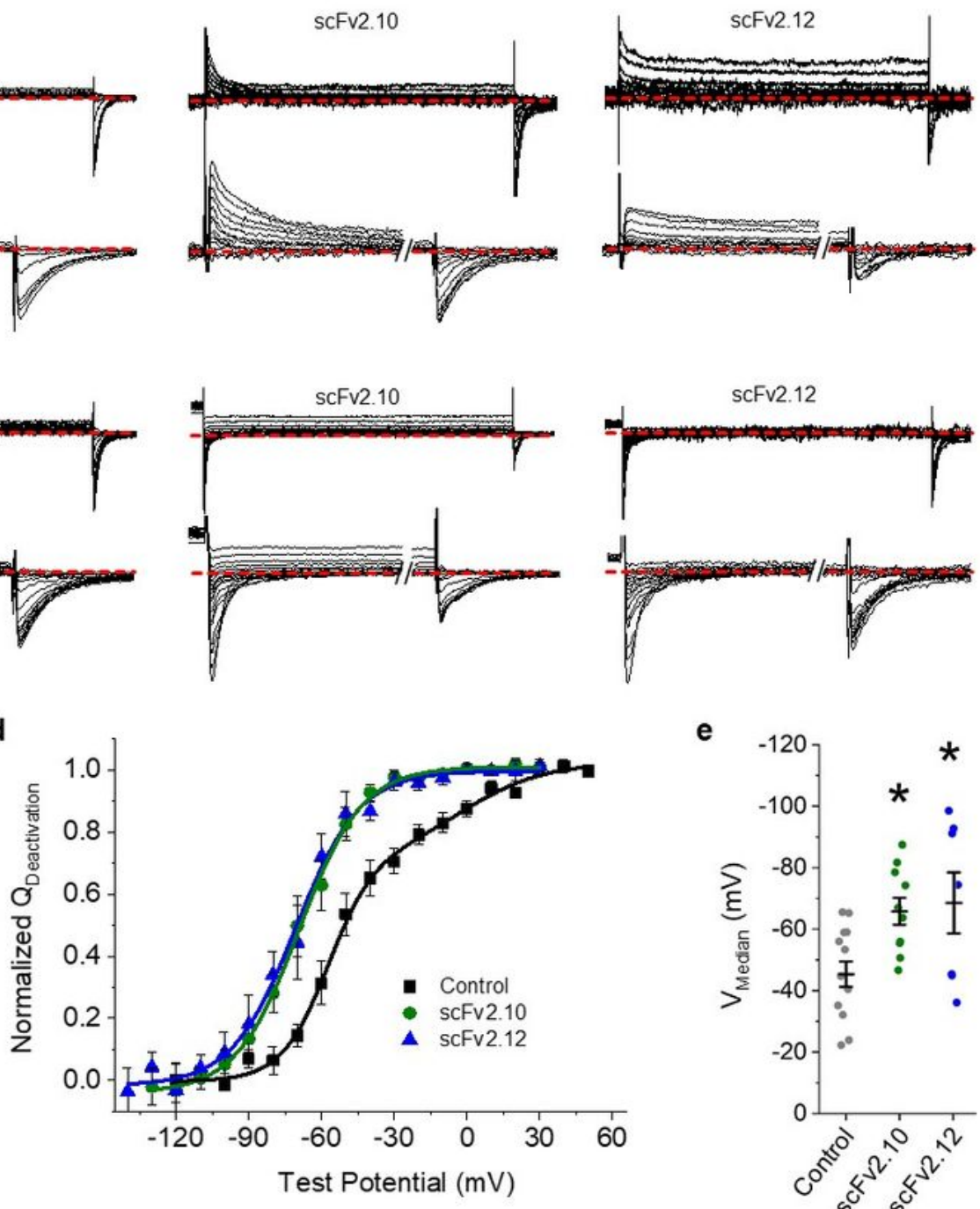

ScFv2.12

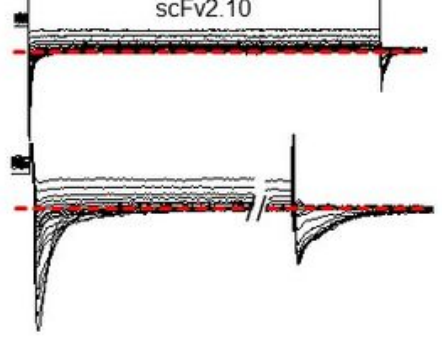

e

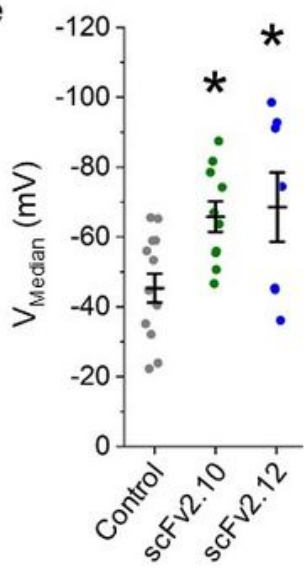

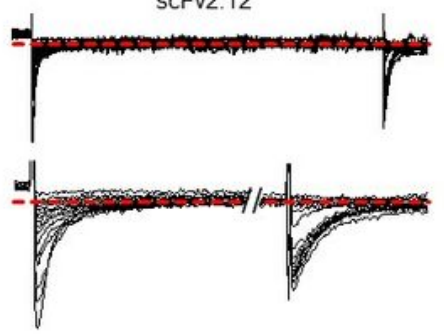

\section{Figure 6}

Anti-PAS antibodies transform hERG 1a gating charge. (a) The pulse protocol (left) and corresponding activation gating current traces for vehicle controls and in the presence of either $10 \mu \mathrm{M}$ scFv2.10 (middle right) or $10 \mu \mathrm{M}$ scFv2.12 (right). hERG 1a gating charge activation was measured by recording gating currents elicited by depolarization from a negative $(-100 \mathrm{mV})$ holding potential. (b) The pulse protocol (left) and corresponding deactivation gating current traces for vehicle controls and in the presence of 
either $10 \mu \mathrm{M}$ scFv2.10 or $10 \mu \mathrm{M}$ scFv2.12. hERG 1a gating charge deactivation was measured by recording gating currents elicited by hyperpolarization from a positive $(+50 \mathrm{mV})$ holding potential. (c) Voltage dependence of hERG 1a voltage sensor activation from vehicle controls (black squares) or in the

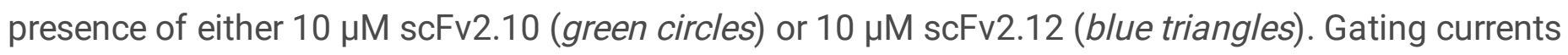
elicited during the test pulse were integrated, and the resultant gating charge was normalized, plotted as a function of test potential and fitted with a Boltzmann function. (d) scFv antibodies hyperpolarize voltage sensor deactivation. Voltage dependence of hERG 1a voltage sensor deactivation from vehicle controls (black squares) or in the presence of either $10 \mu \mathrm{M}$ scFv2.10 (green circles) or $10 \mu \mathrm{M}$ scFv2.12 (blue triangles). Gating currents elicited during the test pulse were integrated, and the resultant gating charge was normalized, plotted as a function of test potential and fitted with either a single Boltzmann (scFv2.10 \& scFv2.12) or a double Boltzmann (control) function. (e) Bar graph depicting the mean $\mathrm{V}_{\text {median }}$ of deactivation for control $(n=12)$, scFv2.10 $(n=14)$, and scFv2.12 $(n=6)$. Both scFv antibodies significantly hyperpolarized the $\mathrm{V}_{\text {median }}$ of VSD deactivation. All data are reported as mean \pm SEM. * indicates $p<0.05$. 
a

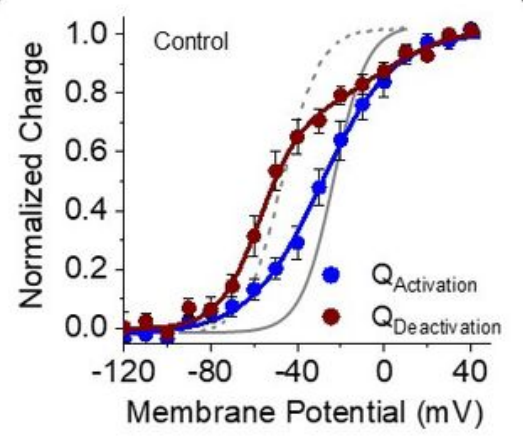

C

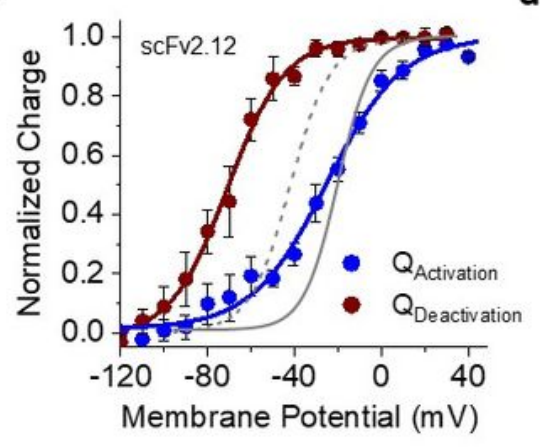

b

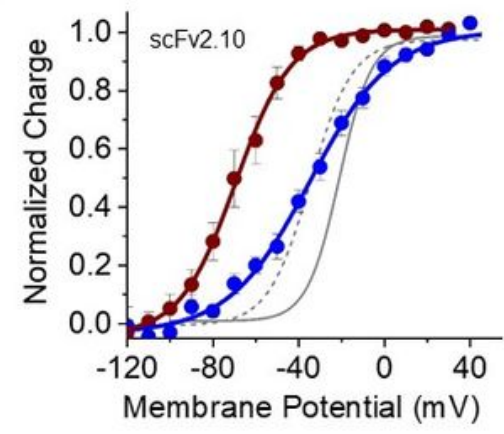

d

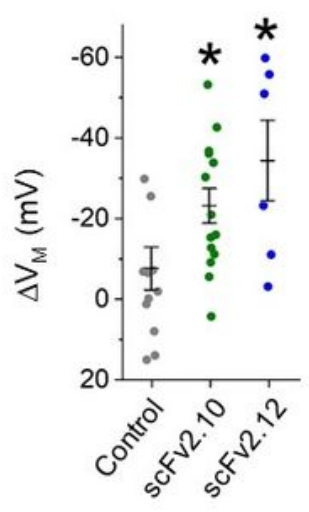

\section{Figure 7}

Anti-PAS antibodies enhance VSD hysteresis. (a-c) Voltage dependence of hERG 1a voltage sensor activation (blue) and deactivation (red) for vehicle controls (a), scFv2.10 (b), and scFv2.12 (c). Fits from ionic currents are shown in gray. Gating currents elicited during the test pulse were integrated, and the resultant gating charge was normalized, plotted as a function of test potential and fitted with a Boltzmann function. (d) The magnitude of hysteresis was quantified by subtracting the $V_{\text {median }}$ of 
deactivation from the $\mathrm{V}_{\text {median }}$ of activation. Hysteresis was significantly larger in the presence of scFv2.10 ( $n=14)$ or the presence of scFv2.12 $(n=6)$ compared with vehicle control recordings $(n=12)$. All data are reported as mean \pm SEM. * indicates $p<0.05$.

\section{Supplementary Files}

This is a list of supplementary files associated with this preprint. Click to download.

- HysteresisPaperSuppMaterial.docx 